\title{
The accuracy of blood pressure measurement by a smartwatch and a portable health device
}

\author{
Noud van Helmond, Cecilia G. Freeman, Christina Hahnen, Nilanjan Haldar, \\ Jacquelyn N. Hamati, Dylan M. Bard, Vignesh Murali, Geno J. Merli \& Jeffrey I. \\ Joseph
}

To cite this article: Noud van Helmond, Cecilia G. Freeman, Christina Hahnen, Nilanjan Haldar, Jacquelyn N. Hamati, Dylan M. Bard, Vignesh Murali, Geno J. Merli \& Jeffrey I. Joseph (2019) The accuracy of blood pressure measurement by a smartwatch and a portable health device, Hospital Practice, 47:4, 211-215, DOI: 10.1080/21548331.2019.1656991

To link to this article: https://doi.org/10.1080/21548331.2019.1656991

Accepted author version posted online: 19

Aug 2019.

Published online: 26 Aug 2019.

Submit your article to this journal $\pi$

山 Article views: 181

Q View related articles ๘

View Crossmark data $₫$

Citing articles: 3 View citing articles $₫$ 


\title{
The accuracy of blood pressure measurement by a smartwatch and a portable health device
}

\author{
Noud van Helmonda,b, Cecilia G. Freemanc, Christina Hahnen ${ }^{\mathrm{a}, \mathrm{d}}$, Nilanjan Haldarc, Jacquelyn N. Hamatic, \\ Dylan M. Bard'c, Vignesh Muralic, Geno J. Merlie and Jeffrey I. Joseph ${ }^{\mathrm{a}}$
}

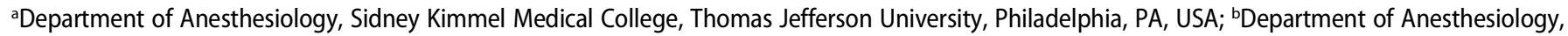
Cooper Medical School of Rowan University, Cooper University Hospital, Camden, NJ, USA; 'Sidney Kimmel Medical College, Thomas Jefferson University, Philadelphia, PA, USA; ${ }^{\mathrm{d} C o l l e g e}$ of Biomedical Sciences, Radboud University, Nijmegen, the Netherlands; ${ }^{\text {Department }}$ of Surgery and Medicine, Sidney Kimmel Medical College, Thomas Jefferson University, Philadelphia, PA, USA
\end{abstract}

\begin{abstract}
Objectives: Handheld medical devices and smartwatches that measure BP without a cuff have recently become available. Since these measurements are relatively more user-friendly than conventional cuff-based measurements they may aid in more frequent BP monitoring. We investigated the accuracy and precision of two popular cuff-less devices: the Everlast smartwatch and the BodiMetrics Performance Monitor.

Methods: We enrolled 127 patients from the Thomas Jefferson University Hospital Preadmission Testing Center. Research staff were trained to measure BP according to manufacturer guidelines for the investigational devices. The Everlast smartwatch provides measurements of systolic (SBP) and diastolic (DBP) BP, whereas the Bodimetrics only provides SBP. Reference BP measurements were obtained using a hospital-grade automated sphygmomanometer. After 5 minutes of quiet sitting, four standard and three investigational BP measurements were taken with sixty seconds in between each measurement. The reference BP value was calculated by determining the average of the 2 standard BP measurements that bounded the investigational measurements. We thus determined 3 comparison pairs for each investigational device in each subject. We calculated the mean (SD) of the absolute difference between the respective investigational devices and the reference for SBP and DBP.

Results: Data from 42 individuals was excluded because of variation in standard BP measurements per prespecified rules. Of 85 participants used for analysis, 36 (42\%) were women, the mean (SD) age was 53 (21) years, $32(38 \%)$ self-reported hypertension, and $97 \%$ of these $(n=31)$ reported taking antihypertensive medications. The average differences between the Everlast watch and reference were 16.9 (13.5) $\mathrm{mm} \mathrm{Hg}$ for SBP and $8.3(6.1) \mathrm{mm} \mathrm{Hg}$ for DBP. The average difference between the Bodimetrics performance monitor and the reference was 5.3 (4.7) $\mathrm{mm} \mathrm{Hg}$ for SBP.

Conclusions: The Everlast smartwatch and the BodiMetrics Performance Monitor we tested are not accurate enough to be used as BP measurement devices.
\end{abstract}

\section{ARTICLE HISTORY}

Received 19 April 2019

Accepted 14 August 2019

\section{KEYWORDS}

Tricorder; smartwatch; m-health; blood pressure measurement; accuracy

\section{Introduction}

Wearable and portable devices that monitor an ambulatory person's health are becoming increasingly popular among consumers. Recently, many manufacturers have been updating their devices to include blood pressure (BP) measurement capabilities using a cuff-less method based on measurements of pulse transit time. Given that hypertension is the leading cause for cardiovascular morbidity and mortality worldwide [1], portable technology that allows consumers to easily measure their BP several times a day would be of great value. However, the convenience that portable health technology provides is not helpful, and even dangerous, if the measurements are inaccurate. Following a protocol derived from the ANSI/AAMI/ISO 2013 standard $[2,3]$ for evaluating non-invasive automated sphygmomanometers, we investigated two popular commercial health devices, the Everlast TR10 fitness watch (Everlast,
New York, NY, USA) and the BodiMetrics Performance Monitor (BodiMetrics, Manhattan Beach, CA, USA), for their accuracy and precision in BP measurements.

\section{Methods}

After obtaining ethical approval from our institutional ethical review board, a sample of 127 ambulatory patients (>18y) was recruited from the Thomas Jefferson University Hospital Preadmission Testing Center. Exclusion criteria were: contraindication for automated BP reading on both arms (e.g. history of breast cancer surgery with radiation or axillary lymph node dissection, arteriovenous fistula for hemodialysis, or open wound), inability to sit up, inability to provide informed consent, pacemaker or other implanted medical device (as per BodiMetrics Performance Monitor manufacturer instructions), irregular heart rhythm (e.g. atrial fibrillation, atrial flutter, or bigeminy) [2], 
missing an upper extremity, hand, or finger, unable to wear a watch due to wrist circumference, or edema of the arm, wrist or hand.

\section{BP measurement devices}

Research staff were trained to measure BP with both of the experimental devices and an FDA approved [4] automated upper arm sphygmomanometer (Cardiocap 5, DatexOhmeda, Madison, WI) according to the manufacturers' guidelines. The Cardiocap 5 is FDA approved based on equivalence to a previously approved BP monitor $(510 \mathrm{k}$ approval). To confirm the Cardiocap's accuracy in BP measurements we compared Cardiocap noninvasive BP measurements to intra-arterial BP measurements from a previously published dataset [5]. The average absolute difference between the two methods from six paired BP measurements in one hundred subjects (582 total pairs) was $4.3 \pm 6.8 \mathrm{~mm}$ $\mathrm{Hg}$ for systolic BP (SBP) and $4.8 \pm 7.2 \mathrm{~mm} \mathrm{Hg}$ for diastolic BP (DBP) measurements. This difference is within recommended limits for accuracy when comparing a non-invasive monitor to intra-arterial measurements [2].

The BodiMetrics Performance Monitor is a commercially available handheld medical device that is sold by several major US-based retailers such as Walmart, Amazon, and Costco. It provides measurements of SBP, but not DBP. Prior to first use, a profile needs to be created by inputting sex, date of birth, height and weight, and an initial calibration for SBP obtained with a conventional upper-arm sphygmomanometer. The device uses visual and auditory instructions to guide users through a measurement; the right index finger needs to be placed beneath the cap on the top of the device, the right thumb on the electrode on the front and the right middle finger on the electrode on the back of the device (Figure 1). Then, the electrode on the left lateral side needs to be placed in the left palm. To ensure a successful measurement, contact needs to be maintained with all electrodes, while the index finger is inserted under the cap. A measurement takes about 30 seconds to complete. SBP is

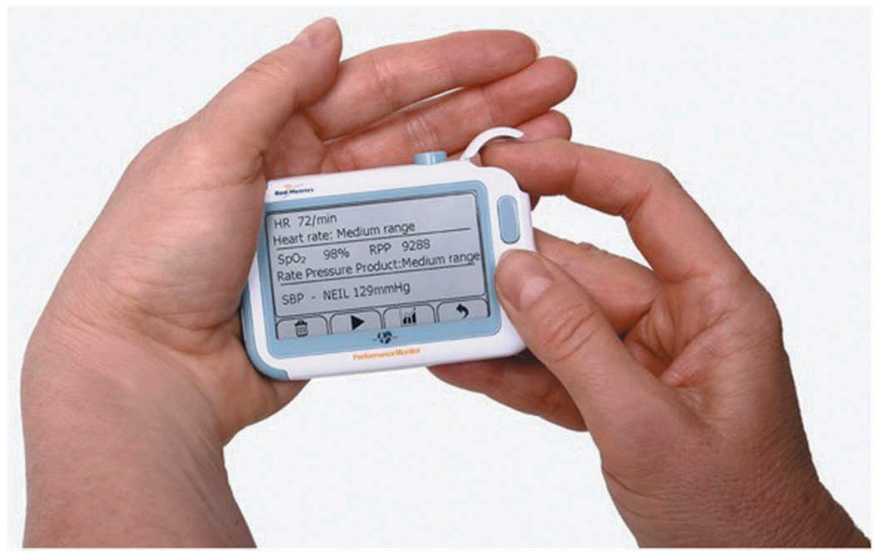

Figure 1. BodiMetrics performance monitor (BodiMetrics, Manhattan Beach, CA, USA).

Systolic blood pressure measurements are performed by placing the right index finger on the plethysmography sensor in the right upper corner under the flap. Additionally, contact has to be made with the ECG electrodes at the front, left lateral side, and back using both hands. measured through the determination of pulse transit time from ECG and photoplethysmography signals [6].

The Everlast TR10 smartwatch (Figure 2, Everlast, New York, NY, USA) is a smartwatch that is for sale through several US-based retailers such as Walmart and Amazon. Unlike the BodiMetrics monitor, the Everlast smartwatch does not require any user specific information or a calibration prior to use. It provides measurements of SBP and DBP. We were unable to confirm the underlying measurement methods through the manufacturer, but the back plate of the watch contains contact electrodes and a photoplethysmography sensor which are likely utilized for the measurement of BP through pulse transit time. A measurement is initiated by clicking the side button and takes about 20 seconds to complete.

\section{Study protocol}

After five minutes of quiet sitting, an initial calibration measurement was taken in line with manufacturer guidelines for the BodiMetrics Performance Monitor. Following the calibration, seven sequential measurements were taken with four from the standard device alternating with three measurements by the investigational devices. Each investigational BP measurement was therefore bounded by two standard measurements. The reference BP value was calculated by determining the average of the two standard BP measurements that bounded the investigational measurements. This yielded three reference-investigational comparison pairs for each device. We calculated the mean (SD) of the absolute difference between the respective investigational devices and the reference for SBP and DBP.

Data from a participant in which any two standard SBP measurements differed by more than $12 \mathrm{mmHg}$ or any two standard DBP measurements differed by more than $8 \mathrm{mmHg}$ were excluded. After taking into account this exclusion criterion, data from 85 patients were used for final analysis [2].

\section{Results}

Of 85 participants, 36 (42\%) were women. The mean (SD) age was 53 (21) years, body mass index was $28(7) \mathrm{kg} / \mathrm{m}^{2} ; 32(38 \%)$ self-reported hypertension, and $97 \%$ of these $(n=31)$ reported taking antihypertensive medications - Table 1. BodiMetrics Performance Monitor measurements correlated well with reference measurements ( $\rho=0.88, P<0.01$ ), whereas Everlast watch measurements did not (Figure 3 ). The average absolute differences between the Everlast watch and reference were 16.9 (13.5) $\mathrm{mm} \mathrm{Hg}$ for SBP and 8.3 (6.1) $\mathrm{mm} \mathrm{Hg}$ for DBP (Figure 4). The average absolute difference between the BodiMetrics Performance Monitor and the reference was 5.3 (4.7) $\mathrm{mm} \mathrm{Hg}$ for systolic BP. The difference between the Everlast watch and reference measurement was dependent on the SBP value, such that lower SBPs were estimated higher and higher SBPs were estimated lower $(\rho=-0.45, P<0.01$, Figure 4). The BodiMetrics Performance Monitor measured a hypertensive BP value ( $\geq 140 \mathrm{~mm} \mathrm{Hg}$ ) for $80 \%$ of the hypertensive reference SBP measurements, whereas the Everlast 

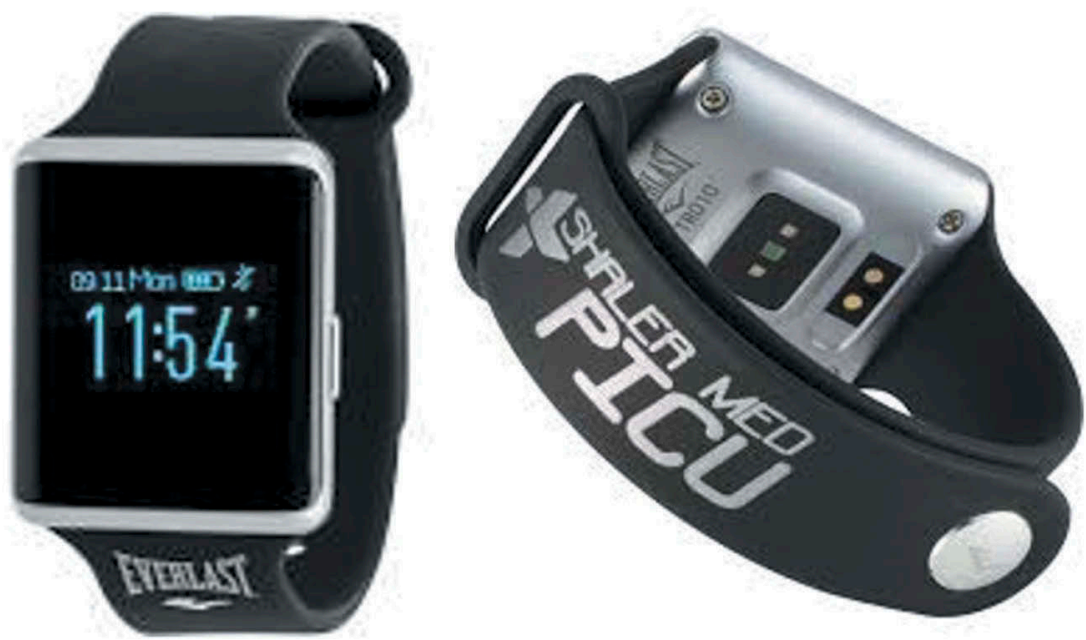

Figure 2. Everlast smartwatch (Everlast, New York, NY, USA).

To enable a blood pressure measurement, the watch has to be worn on the bare wrist making contact with the skin. Systolic and diastolic blood pressure measurements can be started by pushing the button on the right side of the watch. Sensors on the back plate are used for the measurement of blood pressure.

Table 1. Participant characteristics $(\mathrm{N}=85)$.

\begin{tabular}{lc}
\hline Systolic blood pressure in $\mathrm{mm} \mathrm{Hg}$, mean (range) & $126(96-164)$ \\
Diastolic blood pressure in $\mathrm{mm} \mathrm{Hg}$, mean (range) & $78(57-104)$ \\
Age in years (mean $\pm \mathrm{SD}$ ) & $53 \pm 21$ \\
Body mass index in $\mathrm{kg} / \mathrm{m}^{2}$ (mean $\pm \mathrm{SD}$ ) & $28 \pm 7$ \\
Gender, $\mathrm{n}$ male/female (\%) & $49 / 36(58 / 42)$ \\
Ethnicity, $\mathrm{n}(\%)$ & \\
White & $61(72)$ \\
Black & $12(14)$ \\
Asian & $6(7)$ \\
Education, $\mathrm{n}(\%)$ & \\
High school or GED & $28(33)$ \\
College or university degree & $38(45)$ \\
Master's degree & $6(7)$ \\
Doctorate & $5(6)$ \\
Self-reported medical history, $\mathrm{n}$ (\%) & \\
Hypertension & $32(38)$ \\
Taking medication for hypertension & $31(97)$ \\
Diabetes & $13(15)$ \\
Taking medication for diabetes & $12(92)$ \\
Heart attack & $3(4)$ \\
Taking medication for heart attack & $3(100)$ \\
Heart failure & $2(2)$ \\
Taking medication for heart failure & $1(50)$ \\
Peripheral vascular disease & $2(2)$ \\
Taking medication for peripheral vascular disease & $2(100)$ \\
Stroke & $1(1)$ \\
Taking medication for stroke & $0(0)$ \\
Smoking & $8(9)$ \\
\hline
\end{tabular}

watch measured no hypertensive BP values for any of the hypertensive reference SBP or DBP measurements (Figure 3).

\section{Discussion}

The Everlast fitness watch is not accurate enough to be used as BP measurement device [2]. Although the Bodimetrics device was more accurate, possibly due to calibration immediately prior to the study, the BP device still failed to meet accuracy guidelines [2]. Importantly, the Association for the Advancement of Medical
Instrumentation validation protocol [2], from which we derived our investigation, was developed to validate noninvasive cuff-type BP monitors and is based on an underlying assumption that the tested device is capable of measuring BP without an initial calibration reference measurement. Since the calibration measurement for cuff-less monitors is typically $[6,7]$ completed just prior to the conventional static validation protocol, it is unlikely that significant variation in BP from this calibration measurement occurs during a validation series within a study subject. In order for a cuff-less BP monitor to be safe for consumer use it should operate accurately over a range of pressure after being calibrated at a certain pressure, but this concept is not tested when using current validation protocols. The current protocols also do not assess whether calibration of cuff-less devices remains appropriate after a significant time period has passed since initial calibration. Since both devices failed to meet conventional accuracy requirements in our measurement series under static conditions, it seems highly unlikely that they would perform better either at a range of blood pressures different from the calibration value, or after a significant time period since initial calibration.

Widespread use of these devices likely results in the misclassification of patients with hypertension as normotensive, potentially resulting in a delayed diagnosis of hypertension or incorrect self-medication adjustment by patients with known hypertension. Neither one of the devices in our study are approved by the Food and Drug Administration. There are currently at least a dozen similar cuff-less BP measurement devices for sale through US retailers such as Walmart, Amazon, and eBay. The continued sale of these devices without the required validation [8] of safety and efficacy is a public health concern that should be addressed by regulatory bodies, physicians, and patient organizations in order to avoid the potential serious consequences of inaccurate BP measurements. 


\section{a SBP BodiMetrics}

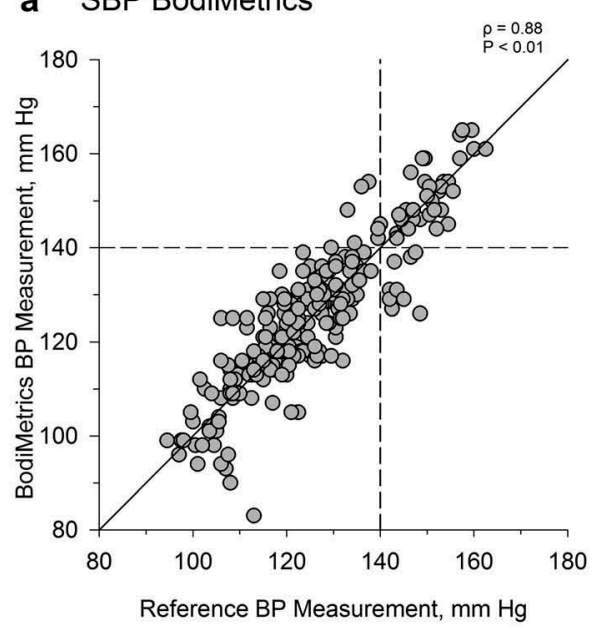

\section{b SBP Everlast}

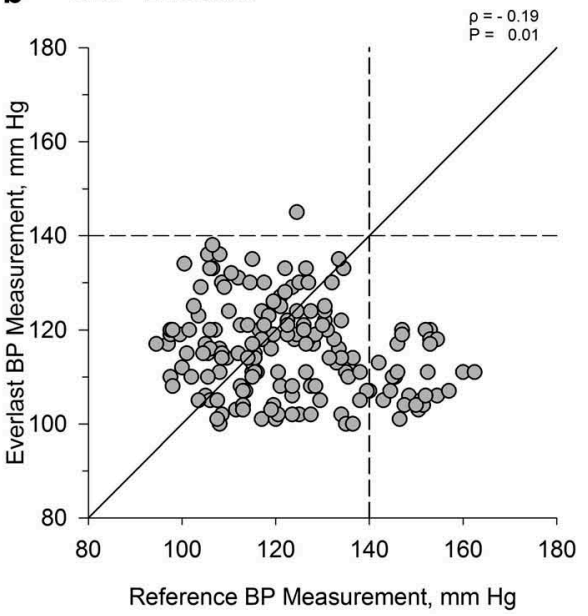

c DBP Everlast

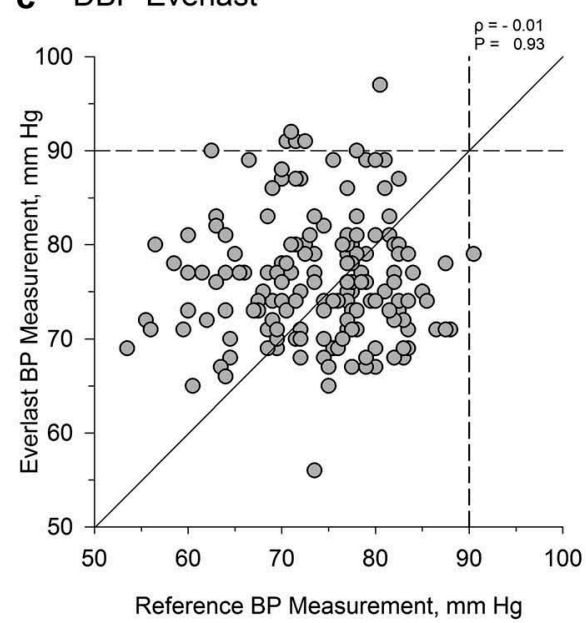

Figure 3. Scatterplots of the bodimetrics performance monitor, everlast watch, and the reference blood pressure measurement.

The solid lines represent the line of identity. The dashed lines represent the cutoff points for stage 2 hypertension. Neither BodiMetrics Performance Monitor residuals nor Everlast watch residuals were normally distributed (Shapiro-Wilk test). Spearman correlation showed a correlation between BodiMetrics SBP measurements and reference measurements, whereas Everlast SBP and DBP measurements were not correlated with reference measurements. SBP $=$ systolic blood pressure, DBP $=$ diastolic blood pressure.

\section{a SBP BodiMetrics}

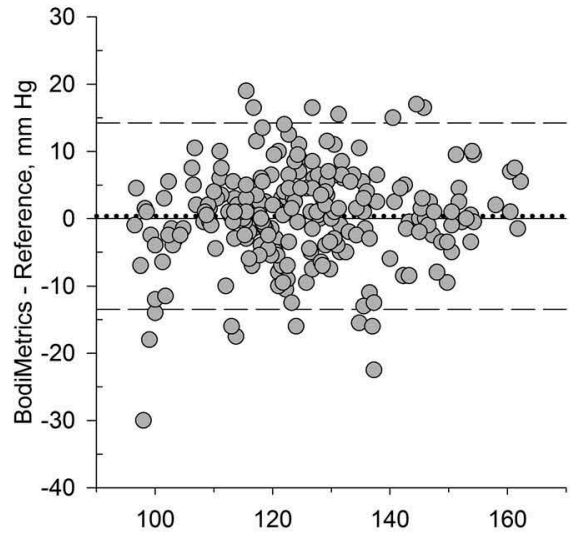

Average of BodiMetrics and Reference, $\mathrm{mm} \mathrm{Hg}$ b SBP Everlast

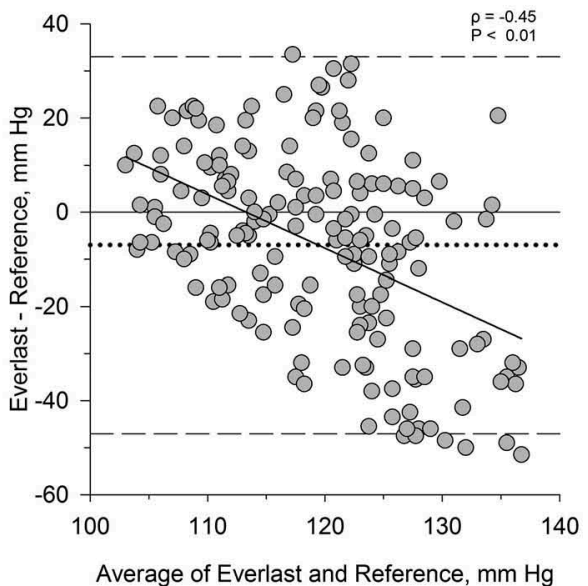

c DBP Everlast

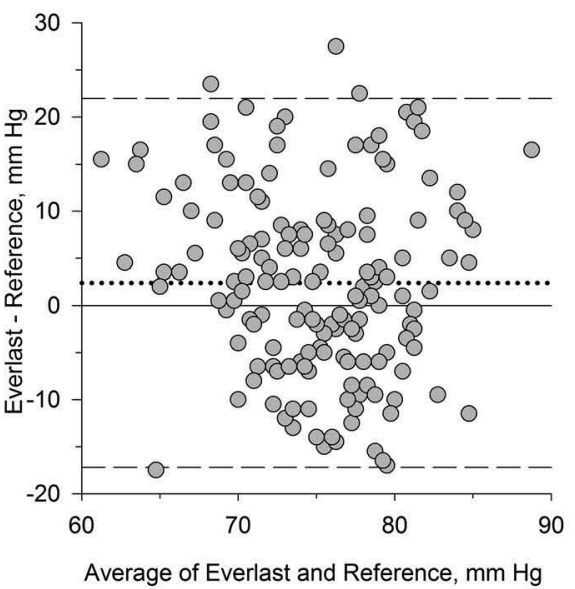

Figure 4. Bland-Altman plots for the bodimetrics performance monitor, everlast watch, and the reference blood pressure measurement.

The dotted lines represent the mean relative differences (investigational minus reference), the dashed lines represent \pm 1.96 SDs for the mean absolute difference. Spearman regression revealed that the difference between the Everlast SBP measurement and reference SBP measurement was dependent on the blood pressure value (residuals were non-normally distributed by the Shapiro-Wilk test). SBP = systolic blood pressure, DBP = diastolic blood pressure.

\section{Conclusion}

The Everlast smartwatch and the BodiMetrics Performance Monitor we tested are not accurate enough to be used as BP measurement devices. The continued sale of these, and similar, non-FDA-approved cuff-less BP measurement devices is a public health concern that should be addressed.

\section{Acknowledgments}

The authors thank Dr. Lashmi Venkatraghavan, Director of Neuroanesthesia, Anesthesia at Toronto Western Hospital, for sharing a deidentified data set of a previously published study [5].

\section{Author contributions}

$\mathrm{N}$ van Helmond helped manage the literature searches, summarize previous related work, devise the study, collect data, analyze data, and write the manuscript. C Freeman, N Haldar, J Hamati, V Murli, and $\mathrm{D}$ Bard helped collect data and edit and revise the manuscript. C Hahnen helped collect data, analyze data, create figures, and edit and revise the manuscript. G Merli helped edit and revise the manuscript. J Joseph helped devise the study and edit and revise the manuscript.

\section{Funding}

This paper was not funded. 


\section{Declaration of interest}

$\mathrm{J}$ Joseph is a founder, equity owner, and has received research support from RTM Vital Signs, LLC, a company developing a non-invasive and long-term implantable vital sign monitoring system with real-time diagnostic algorithms and data transferred via cell phone to a central monitoring system. The authors have no other relevant affiliations or financial involvement with any organization or entity with a financial interest in or financial conflict with the subject matter or materials discussed in the manuscript apart from those disclosed.

\section{Reviewer disclosures}

Peer reviewers on this manuscript have no relevant financial or other relationships to disclose.

\section{References}

1. GBD 2015 Risk Factors Collaborators. Global, regional, and national comparative risk assessment of 79 behavioural, environmental and occupational, and metabolic risks or clusters of risks, 1990-2015: a systematic analysis for the global burden of disease study 2015. Lancet. 2016;388(10053):1659-1724.
2. Graves JW, Quinn D. Noninvasive sphygmomanometers, Part 2: clinical investigation of automated measurement type. Vols. 81060-2. Geneva, Switzerland: ANSI/AAMI/ISO; 2013.

3. Plante TB, Urrea B, MacFarlane ZT, et al. Validation of the instant blood pressure smartphone app. JAMA Intern Med. 2016;176(5):700-702.

4. US Food and Drug Administration. Datex Ohmeda Cardiocap 5. 2001. cited 201801 11. Available from: https://www.access data.fda.gov/cdrh_docs/pdf/K012837.pdf

5. Sheshadri V, Tiwari AK, Nagappa M, et al. Accuracy in blood pressure monitoring: the effect of noninvasive blood pressure cuff inflation on intra-arterial blood pressure values. Anesth Essays Res. 2017;11(1):169-173.

6. Schoot TS, Weenk M, van de Belt TH, et al. A new cuffless device for measuring blood pressure: a real-life validation study. J Med Internet Res. 2016 May 5;18(5):e85.

7. Boubouchairopoulou N, Kollias A, Chiu B, et al. A novel cuffless device for self-measurement of blood pressure: concept, performance and clinical validation. J Hum Hypertens. 2017 Jul;31 (7):479-482.

8. US Food and Drug Administration - Non-Invasive Blood Pressure (NIBP) Monitor Guidance. 1997. cited 20180111. Available from: https://www.fda.gov/Regulatorylnformation/ Guidances/ucm080219.htm 